Meta

Journal des traducteurs

Translators' Journal

\title{
La terminologie de l'avenir : un dialogue Homo Sentiens-Machina Sapiens
}

\section{Silvia Pavel}

Volume 32, numéro 2, juin 1987

Vers l'an 2000. La terminotique, bilan et prospectives

Objectives: Year 2000 Terminotics. State of the Art, Prospects for the

Future

URI : https://id.erudit.org/iderudit/003804ar

DOI : https://doi.org/10.7202/003804ar

Aller au sommaire du numéro

Éditeur(s)

Les Presses de l'Université de Montréal

ISSN

0026-0452 (imprimé)

1492-1421 (numérique)

Découvrir la revue

Citer cet article

Pavel, S. (1987). La terminologie de l'avenir : un dialogue Homo

Sentiens-Machina Sapiens. Meta, 32(2), 124-129.

https://doi.org/10.7202/003804ar d'utilisation que vous pouvez consulter en ligne.

https://apropos.erudit.org/fr/usagers/politique-dutilisation/ 


\section{LA TERMINOLOGIE DE L'AVENIR : UN DIALOGUE HOMO SENTIENS-MACHINA SAPIENS}

Silvia Pavel

Direction de la terminologie, Secrétariat d'État, Ottawa, Canada

Depuis le lancement du premier spoutnik, il y a trente ans, la planétarisation des communications par satellite marque la transition de la société industrielle axée sur le capital et le travail, à la société postindustrielle dont la principale ressource stratégique est l'information et l'expertise. Si la restructuration postmoderne s'est opérée en quelques décennies, le passage de la société agraire à la société industrielle avait duré un siècle. Le rythme et la nature même des changements invitaient à puiser dans l'expérience du passé pour mieux comprendre et façonner le présent. Mais aujourd'hui nous ne pouvons plus vivre "l'œil rivé au rétroviseur " (de Closets 1985). Les changements se succèdent si vite qu'on n'a presque pas le temps de réagir. Il nous faut plutôt "préfigurer l'avenir " (Bell 1979) tout en évaluant et en façonnant le présent.

La prospective économique, politique et culturelle est déjà bien entamée. L'on ne pouvait tarder de l'envisager dans une discipline telle que la terminologie ${ }^{1}$ dont le rôle véhiculaire ne cesse de s'affermir depuis la parution des ouvrages de $\mathbf{E}$. Wüster (Autriche) et de D.S. Lotte (Union soviétique) en 1931.

\section{LA TERMINOLOGIE : UNE DISCIPLINE, UN MÉTIER}

La terminologie se définit comme

la pierre angulaire de la systématique des connaissances (théorie générale de la science, classification des notions par discipline scientifique), du transfert des connaissances, des techniques et de la technologie (enseignement, formation et perfectionnement), du transfert de l'information d'une langue à l'autre (traduction et interprétation) et de la production de l'information dans tout domaine du savoir (H. Felber 1982).

Dans ce cadre théorique, le praticien de la terminologie recherche et étudie systématiquement la littérature technoscientifique de sa spécialité en dégageant le réseau de notions qui la sous-tendent. Des documentalistes l'aident à se procurer la documentation, des experts l'aident à structurer ses connaissances en hiérarchies et hétérarchies conceptuelles.

L'étude sémantique des notions figurant aux nœuds de ces structures arborescentes lui permet de les définir en corrélation et d'en regrouper les dénominations (termes) repérées dans la documentation spécialisée. L'étude linguistique des termes détermine leurs caractéristiques formelles, en décrit l'usage et parfois le prescrit.

Ces données sémantiques et grammaticales sont consignées sur fiches uninotionnelles dont l'ensemble constitue la nomenclature à partir de laquelle l'on crée des vocabulaires. En terminologie comparée, des nomenclatures parallèles basées sur des textes en deux ou plusieurs langues servent à la création de vocabulaires bilingues ou multilingues. 


\section{RÉTROSPECTIVE TERMINOGRAPHIQUE : LA PREMIÈRE ÈRE INFORMATIQUE}

Les grandes étapes de l'activité terminologique, à savoir : documentation, étude notionnelle, consignation et diffusion, sont essentiellement les mêmes depuis cinquante ans quels que soient le pays, la spécialité ou la langue. Par contre, les méthodes et les moyens utilisés revêtent une grande diversité. L'intégration du téléphone, de l'ordinateur et de la télévision au traitement de l'information a accentué cette diversité des pratiques terminologiques au cours des années 1970-1985, période que l'on qualifie déjà de "première ère informatique" (Feigenbaum, McCorduck 1983) par rapport à une deuxième ère, celle de la cinquième génération d'ordinateurs et de l'intelligence artificielle (1985-2000).

\section{DOCUMENTATION}

Durant cette première ère on assiste à l'informatisation des moyens de collecte, de tri, de classement et de diffusion de la documentation technoscientifique et, depuis l'apparition des banques de données documentaires, à l'accélération exponentielle de la vitesse de circulation de l'information textuelle. Quelques secondes suffisent aujourd'hui pour accéder en mode interactif à des informations que le courrier traditionnel transmettait en plusieurs jours. À cette grande vitesse de circulation s'ajoute le rythme croissant de production de l'information. Ainsi, selon les statistiques fournies par l'économiste John Naisbitt, la production d'articles scientifiques aux États-Unis se situait en 1982 entre 6000 et 7000 articles par jour. Elle s'accroît, depuis, au rythme annuel de $13 \%$ et peut doubler tous les 30 mois. En notant des tendances similaires dans d'autres pays et en englobant dans leur production les autres catégories de textes scientifiques, il serait possible de concevoir les proportions de ce véritable bombardement informationnel.

Dans ces conditions, la réduction du temps d'accès aux bibliographies automatisées exhaustives est directement proportionnelle à l'augmentation du temps nécessaire à l'obtention des ouvrages, à leur lecture et à leur sélection en fonction de critères de fiabilité et de pertinence préétablis. Le terminologue ne dispose pas encore de moyens informatiques adéquats qui privilégient l'accès sélectif à l'information par rapport à sa diffusion indiscriminée.

\section{L'ÉTUDE DES NOTIONS PAR LANGUE DE SPÉCIALITÉ2 2}

L'impact de l'informatisation sur cette étape de la recherche terminologique est considérable dans les disciplines classiques où, grâce justement à l'accessibilité de l'information, le terminologue peut profiter du travail de ses prédécesseurs et collègues en matière de classification et définition des notions, lors de la mise à jour d'un système notionnel.

L'on ne peut en dire autant des notions appartenant à des domaines de pointe, comme la biotechnologie ou l'intelligence artificielle, où quasiment chaque école de pensée crée sa propre terminologie et son propre réseau sémantique, et où la classification des connaissances reste à faire.

Dans les deux cas, les processus d'analyse des notions, de leur définition, ainsi que l'étude linguistique de leurs dénominations n'ont pas encore été automatisés malgré quelques études de faisabilité envisageant la génération automatique de définitions et la classification informatisée des notions en fonction de traits sémantiques et rapports logiques. Il n'est d'ailleurs pas sans intérêt de remarquer que même en intelligence artificielle, l'étude des notions dans une langue de spécialité - étape première de la construction de tout système expert - reste l'apanage de l'homme (en l'occurrence, de l'ingénieur cogniticien qui en établit le vocabulaire avec le spécialiste de la discipline concernée). 


\section{L'ENREGISTREMENT DES DONNÉES}

Un saut qualitatif décisif dans la dialectique de la recherche fut réalisé vers la fin des années 60 par l'introduction du traitement informatique des données terminologiques : l'accès à l'information terminologique devint simple et rapide, les facilités de mise à jour mirent l'activité terminologique au rythme du progrès des connaissances, et augmentèrent l'efficacité et l'exactitude du travail terminographique.

Adoptées par plusieurs organismes à vocation linguistique, les premières banquesdictionnaires contribuèrent à améliorer la qualité des traductions technoscientifiques. D'autres banques, orientées davantage vers le traitement et la diffusion des terminologies normalisées jouent déjà un rôle prépondérant dans l'uniformisation de l'usage terminologique, favorisent les transferts terminologiques, ou deviennent les outils indispensables de l'aménagement linguistique.

Durant les dix dernières années et surtout depuis l'avènement de la microinformatique, plusieurs instituts de recherche, universités et entreprises se sont dotés de minibanques spécialisées dans divers domaines du savoir et de la pratique en essayant de répondre aux besoins de catégories spécifiques d'utilisateurs. La grande diversité des matériels et des logiciels utilisés par toutes ces banques a entraîné la différenciation des principes de consignation des données, des méthodes de saisie, de stockage et de mise à jour.

Il s'agit maintenant de créer les interfaces capables d'assurer la compatibilité des équipements et de s'entendre sur un format de consignation qui facilite les échanges terminologiques interbanques.

\section{LA DIFFUSION DE L'INFORMATION TERMINOLOGIQUE}

Le traitement informatique permet une grande centralisation de l'information terminologique et sa constante mise à jour. Il rend possible la diffusion massive de cette information par des terminaux d'abonnés aux banques de données, par des publications classiques (glossaires, lexiques, cahiers de néologie, vocabulaires normalisés, dictionnaires, etc.) ou encore par l'entremise des services de renseignements téléphoniques créés auprès des organismes à vocation terminologique.

Grâce à l'ordinateur, les progrès réalisés en matière de diffusion n'ont pas de précédent dans l'histoire de la terminologie : une grande banque de terminologie peut servir en direct plusieurs milliers d'utilisateurs, dont certains situés à des distances transatlantiques ; mettre à jour son contenu au rythme de quelques milliers de fiches par semaine, en extraire des publications à l'intention d'autres utilisateurs, fournir des dizaines de milliers de renseignements téléphoniques, échanger des informations et de l'expertise avec de nombreux organismes et contribuer efficacement aux efforts de normalisation

Mais déjà, de nouveaux besoins surgissent : l'élargissement de l'accès simultané en direct et l'ajout du micro-ordinateur à l'environnement bureautique du traducteur, la modularisation de la saisie, l'augmentation des apports terminologiques des abonnés et la stimulation des recherches coopératives, où encore l'intégration du savoir terminologique à la formation des futurs spécialistes, le développement des terminologies dans les langues du tiers-monde, la dissémination de nouveaux concepts technoscientifiques et la création de néologismes présentant des caractéristiques formelles communes aux langues d'une même famille.

De quels moyens techniques devrait-on se doter afin de satisfaire à ces besoins ?

\section{PERSPECTIVES DU DIALOGUE HOMME-MACHINE : LA DEUXIÈME ÈRE INFORMATIQUE}

Dans un premier temps, il faudrait optimiser les performances des équipements en place : augmenter leur puissance de traitement et de mémoire ; inclure l'accès multiban- 
ques, le courrier électronique et le traitement de texte à la traduction assistée par ordinateur ; créer des interfaces de communication indépendantes des machines, des interfaces qui généralisent le dialogue homme-machine et machine-machine en temps réel et à peu de frais; développer le dialogue en langage naturel qui épargne à l'utilisateur non informaticien l'apprentissage de plusieurs langages d'interrogation pour les différentes banques consultées; se raccorder à des serveurs installés dans des villes et des pays différents.

Dans un deuxième temps, il faudrait intégrer aux systèmes existants des programmes capables de trier et de sélectionner les informations en simulant le raisonnement humain. De tels programmes existent déjà dans plusieurs domaines d'activité : ce sont les systèmes experts.

\section{TERMINOLOGIE ET INTELLIGENCE ARTIFICIELLE}

Les systèmes experts sont des ensembles logiciels structurant des connaissances explicites relatives à un domaine particulier pour offrir un comportement comparable à celui de l'expert humain. L'utilisateur de ces " assistants intelligents " (Bonnet 1983) y trouve des aides au transfert d'expertise (diagnostic médical, planification financière, prospection géologique, détection de pannes); des aides à la traduction automatique de textes techniques ; des aides à la rédaction (correspondance d'affaires, correction des erreurs d'orthographe et de grammaire, rephrasage de textes) ; des aides documentaires (analyse de texte et production de résumés permettant à des lecteurs potentiels de savoir rapidement si un texte les intéresse); ou des aides pédagogiques (formation à une technique particulière).

Une énumération aussi sommaire que celle-ci des aspects utilitaires des systèmes experts existant déjà sur le marché ne manque pas de souligner la pertinence de la terminologie telle que définie par $\mathbf{H}$. Felber à l'ère de l'intelligence artificielle. En effet, chacune de ces applications fait appel, à des degrés divers, à l'expertise terminologique, que ce soit sous la forme de la structuration des connaissances, du transfert pédagogique, de la traduction, ou de la production de documents spécialisés. Il ne reste qu'à impliquer les terminologues.

Il serait aussi intéressant d'explorer en contrepartie les applications possibles du génie cognitif à la recherche terminologique proprement dite. Ainsi, à l'étape de la documentation, le travail du terminologue serait grandement simplifié s'il disposait de résumés générés automatiquement suite à la reconnaissance optique des textes sélectionnés intelligemment dans les meilleures bibliothèques. Le dépouillement par lecture et impression optique réduirait considérablement le fastidieux de la consignation manuscrite. L'accès à la partie vocabulaire de plusieurs systèmes experts dans un domaine partículier compenserait ou compléterait les conseils d'un expert humain à l'étape de la structuration notionnelle, tandis que des systèmes experts en traitement de texte prendraient en charge la rédaction de fiches terminologiques. Le terminologue serait en mesure de manipuler efficacement une bien plus grande quantité d'informations et de mieux exercer son esprit de discernement et sa compétence linguistique lors de l'établissement ou de la mise à jour des terminologies.

En cas de doute devant des cas litigieux il pourrait toujours avoir l'avis d'un système expert en théorie de la terminologie. Quant à l'apparition de notions nouvelles dans sa spécialité, le terminologue serait parmi les premiers au courant de leurs origines, de leurs significations et des termes choisis par leurs inventeurs pour les désigner. Ces derniers ne verraient peut-être plus d'inconvénient à consulter un terminologue avant de baptiser leur invention, puisqu'ils sauraient quelles banques possèdent le répertoire des formants lexicaux et des règles lexicales de la langue en question, ainsi que le répertoire de tous les néologismes du domaine. En rédigeant des articles, les spécialistes pour- 
raient consulter de telles banques afin d'éviter les emprunts ad hoc du genre "la méthode dite du Best Few" ou "le modèle du blackboard" lorsqu'ils se réfèrent à des notions venues d'ailleurs... Ils pourraient du reste les consulter d'ores et déjà, avec de bonnes chances d'y trouver une solution satisfaisante.

Que retiendra la terminologie des possibilités ouvertes par l'intelligence artificielle? Il est difficile de le prévoir sans considérer au préalable ce qu'elles coûteront en termes de ressources matérielles et humaines par rapport à ce qu'elles apporteront à notre métier en termes de progrès réellement nécessaire. Entre-temps, rien n'interdit d'espérer qu'en terminologie, comme en toute chose, la sensibilité intellectuelle de l'homo sentiens décidera des usages du savoir et non pas l'inverse.

\section{PRÉVISION ET SCIENCE FICTION}

Excellente gymnastique de l'esprit, l'exploration du possible ne peut toutefois pas faire oublier les acquis terminologiques, ni les limites des systèmes experts actuels. Car, aussi utiles qu'ils soient, ces " raisonneurs informatisés " ne possèdent pas de vraie intelligence. Ils ne peuvent pas apprendre ni s'améliorer en se basant sur leur expérience. $\mathrm{Ni}$ montrer du bon sens. Ni réagir à des situations nouvelles de manière non programmée. Dans dix ou vingt ans peut-être, les recherches en intelligence artificielle aboutiront à la conception de systèmes experts dotés de capacités cognitives plus semblables à celles de l'homme : apprendre et évoluer grâce à ses expériences ; comparer les expériences présentes aux expériences passées en leur trouvant des relations significatives; se donner des nouvelles informations et arriver à ses propres conclusions; expliquer les raisons de ses jugements et identifier les processus de pensée sous-jacents.

Les ordinateurs cognitifs (Schank 1984) - domaine privilégié des recherches théoriques en intelligence artificielle - seront capables d'entendre, de comprendre et de parler le langage naturel, et de reconnaître l'écriture, même manuscrite. Ils s'instruiront en assimilant de nouvelles connaissances à partir de sources orales ou écrites. Ils pourront restructurer leurs représentations conceptuelles en passant ces nouvelles acquisitions au filtre d'une mémoire critique, sachant éviter les erreurs du passé.

En cette fin de siècle, l'on peut anticiper avec vraisemblance l'arrivée des télécommunications sans fil, des bases de connaissances à la portée de tous, et des robots à tout faire dans la société du télétravail, de la maison électronique et du collège invisible. Mais prédire toutes les applications et les performances possibles des machines pensantes autodidactes est un exercice de l'imagination que même la communauté de l'intelligence artificielle préfêre laisser aux soins de la science fiction (la Recherche 1985).

C'est d'ailleurs rendre à César ce qui est à César car, depuis les statues animées de Héphaïstos aux océans-cerveaux de Solaris, l'intelligence - humaine, artificielle, ou extra-terrestre - s'inscrit parmi les thèmes fabuleux du récit mythique et de la science fiction.

Quant à la terminologie, même si elle ne deviendra jamais un sujet littéraire, on se console en la voyant pratiquée avec délectation par tant d'écrivains qui créent des milliers de néologismes fictifs pour désigner les nombreux êtres et objets peuplant les mondes possibles. Et, qui sait ? Le jour viendra peut-être où spécialistes et terminologues découvriront dans la créativité lexicale de la littérature, de la publicité et des argots, des nouvelles sources d'inspiration pour nommer la multitude d'inventions techniques et scientifiques du monde réel.

\section{Notes}

1. Deux colloques récents ont permis d'amorcer ce processus : le premier, "L'ère nouvelle de la terminologie "(novembre 1985) fut organisé à Montréal par l'Office de la langue française et la Société des traducteurs du Québec; le deuxième, "Terminologie et technologie nouvelles» (décembre 1985) fut 
organisé à Paris par le Commissariat de la langue française en collaboration avec l'Office de la langue française.

2. Sager et al. (1980:21,182) définit les langues de spécialités comme "means of linguistic communication required for conveying special subject information among specialists of the same subject». Pour une compréhension approfondie du concept, voir Kocourek (1982).

\section{BIBLIOGRAPHIE}

BELL, Daniel (1979) : "Thinking Ahead ", in Harvard Business Review, mai-juin, pp. 20-42.

BONNET, Alain (1983) : l'Intelligence artificielle — promesses et réalités, Paris, Inter-Éditions.

BOUVERESSE, Jacques (1984) : Rationalité et cynisme, Paris, Minuit.

DE CLOSETS, François (1985) : Tous ensemble, Paris, Seuil.

FEIGENBAUM, Edward, Pamela McCORDUCK (1983) : The Fifth Generation, a Signet Book, New American Library.

FELBER, Helmuth (1982) : "Some Basic Issues of Terminology ", in The Incorporated Linguist, vol. 21, n 1 , pp. 12-23.

KOCOUREK, Rostislav (1982) : la Langue française de la technique et de la science, Paris, La documentation française.

LOTTE, D.S. (1931) : " Otcherednyé zatatchi naoutchnoteckhnitcheskoï terminologii " (D’autres tâches de la terminologie scientifique et technique), in Izvestiia ANSSSR, otdelenié tekhnitcheskikh naouk, $\mathrm{n}^{\circ} 7$

NAISBITT, John (1982) : Megatrends, New York, Warner Books Inc.

SAGER, J.C. et al. (1980) : English Special Languages, Wiesbaden.

SCHANK, Roger (1984) : The Cognitive Computer, Addison-Wesley Publishing Co., Inc.

WÜSTER, Eugen (1931) : Internationale Sprachnormung in der Technik (Normalisation internationale de la terminologie technique), Berlin, VDI - Verlag.

"L'intelligence artificielle", numéro spécial de la revue la Recherche, vol. 16, n 170, Paris, 1985. 